\title{
ON ONE ALGORITHM FOR SOLVING THE PROBLEM OF SOURCE FUNCTION RECONSTRUCTION
}

\author{
VYACHESLAV MAKSIMOV \\ Institute of Mathematics and Mechanics \\ Ural Branch of the Russian Academy of Sciences, 16 S. Kovalevskoi Str., Ekaterinburg, 620219 Russia \\ e-mail: maksimov@imm.uran.ru
}

\begin{abstract}
In the paper, the problem of source function reconstruction in a differential equation of the parabolic type is investigated. Using the semigroup representation of trajectories of dynamical systems, we build a finite-step iterative procedure for solving this problem. The algorithm originates from the theory of closed-loop control (the method of extremal shift). At every step of the algorithm, the sum of a quality criterion and a linear penalty term is minimized. This procedure is robust to perturbations in problems data.
\end{abstract}

Keywords: reconstruction, source function, feedback control.

\section{Introduction and problem formulation}

In the theory of differential equations, the following problem is well-known: it is required to determine parameters of a differential equation provided a given function plays the role of its solution. Unknown parameters (generally speaking, not constant and depending on time) can be controls, dynamical disturbances, coefficients, some system characteristics and so on. As a rule, such problems are ill-posed. First, a number of parameter values may correspond to a given solution. This property rather often results in a new problem of choosing parameters. For example, in the problem of control reconstruction, the interest is usually in finding a control with an extremal (maximal or minimal) energy resource. Second, the mapping "solution $\longrightarrow$ parameter" is discontinuous in the general case. Therefore, this mapping cannot be used to approximate a desired parameter in the case when a disturbed solution is given instead of an exact one. If some function, which is not a solution, is given instead of a disturbed solution of an equation, additional difficulties arise. Under such conditions, the "approximative" feature should be provided by some regularizing procedures. Besides, there exists the problem of sufficiently convenient and constructive description and determination of the mapping "solution $\longrightarrow$ parameter". Issues of determining some parameters through equation solutions are often called reconstruction (identification) problems. Recently, methods of solving reconstruction problems have been intensively developed.
A considerable number of works is devoted to solving problems of reconstructing right-hand parts (e.g., a source function) of parabolic equations through results of sensor observations. A typical problem from this field is as follows.

Let us imagine a water reservoir occupying an area $\Omega$ and $n$ contamination sources located in subareas $\Omega_{1}, \ldots, \Omega_{n}$ of $\Omega$. It is assumed that an input concentration rate of some contaminant at every point $\xi$ in the source area $\Omega_{j}$ is modeled as $u_{j}(t) \omega_{j}(\xi)$, where $t$ is current time. The positive function $u_{j}(t)$ is a measure of the timevarying intensity of the source located in $\Omega_{j} ; u_{j}(t)$ represents the current rate of the contamination inflow in $\Omega_{j}$. Let $x(t, \xi)$ be the current concentration of the contaminant at a point $\xi$ in $\Omega$. Some information on the distribution of $x(t, \xi)$ in $\Omega$ is registered by $m$ sensors. The sensors register weighted average concentrations $z_{1}(t), \ldots, z_{m}(t)$ in fixed subareas $\Theta_{1}, \ldots, \Theta_{m}$ of $\Omega$ :

$$
z_{k}(t)=\int_{\Theta_{k}} p_{k}(\xi) x(t, \xi) \mathrm{d} \xi, \quad k=1, \ldots, m,
$$

the positive weight coefficients $p_{k}(\xi)$ are supposed to be given. The problem of reconstructing the righthand part (the intensity reconstruction problem) is as follows. Observing the weighted average concentrations $z_{1}(t), \ldots, z_{m}(t)$ of the contaminant in the areas $\Theta_{1}, \ldots, \Theta_{m}$, reconstruct the intensities of the contamination sources, $u_{1}(t), \ldots, u_{n}(t)$, in the source areas 
$\Omega_{1}, \ldots, \Omega_{m}$. This problem has been studied by many authors (see, e.g., (Omatu and Seinfeld, 1989; Korbicz and Zgurowski, 1991; Uciński, 1999)).

In the present work, a new algorithm for solving such a problem for an abstract parabolic equation is suggested. A particular case of such an equation is a diffusion equation describing the process of contaminant propagation in the atmosphere or liquid media. This algorithm rests upon constructions of the theory of stable dynamical inversion based on the combination of methods of the theory of ill-posed problems and that of positional control. The essence of the technique described in (Blizorukova and Maksimov, 1998; Digas et al., 2003; Kryazhimskii and Osipov, 1987; Kryazhimskii et al., 1997) is that a reconstruction algorithm is represented as a control algorithm for some auxiliary dynamical system. It should be noted that this technique exploits the idea of stabilizing appropriate functionals of the Lyapunov type by means of extremal shift (Krasovskii and Subbotin, 1988). Thus, the technique combines the stabilization principle with that of extremal shift in some scheme of control with a model. In the beginning, some functional treated as a Lyapunov type one is introduced. Then, a control law for an auxiliary system is chosen. This law uses the idea of extremal shift providing a "weak growth" of the functional in time.

Let us pass to the statement of the problem under investigation. In a real Hilbert space $\left(H,|\cdot|_{H}\right)$, the following parabolic equation is considered:

$$
\begin{gathered}
\dot{x}(t)+A x(t)=B u(t)+f(t), \\
t \in T=[0, \vartheta], \quad x(0)=x_{0} \in H .
\end{gathered}
$$

Here, $A: V \rightarrow V^{*}$ is a linear continuous symmetric operator satisfying (for some $c>0$ and $\lambda \in \mathbb{R}$ ) the coercivity condition

$$
\langle A y, y\rangle_{V}+\lambda|y|_{H}^{2} \geq c\|y\|^{2}, \quad \forall y \in V .
$$

$(V,\|\cdot\|)$ is a separable and reflexive Banach space, which is densely and continuously embedded in the space $H$ identified with its conjugate space $\left(H=H^{*}\right) ;\langle\cdot, \cdot\rangle_{V}$ is the duality between $V$ and $V^{*} ; x(t)$ is the phase state of the system (1) at the moment $t ; u(t) \in U$ is a disturbance generating the motion $x(\cdot)$; the space of disturbances $\left(U,|\cdot|_{U}\right)$ is a Hilbert space with a scalar product $(\cdot, \cdot)_{U} ; f(\cdot) \in L_{2}(T ; H)$ is a given input action; $B: U \rightarrow H$ is a linear continuous operator.

A solution of the system (1) (which is understood in the weak sense) is defined to be a unique continuous function $x(\cdot)=x\left(\cdot ; x_{0}, u(\cdot)\right)$ of the form

$x(t)=S(t) x_{0}+\int_{0}^{t} S(t-\tau)(B u(\tau)+f(\tau)) \mathrm{d} \tau, \quad t \in T$.

Here $S(t): H \rightarrow H(t>0)$ is the semigroup of continuous linear operators generated by the operator $A$. As is known (Bensoussan et al., 1992), for all $u(\cdot) \in L_{2}(T ; U)$, $f(\cdot) \in L_{2}(T ; H), x_{0} \in H$, there exists a unique solution of the system (1). Below it is assumed that the set of admissible system inputs $u(\cdot)$ is of the form

$$
U_{*}=\left\{u(\cdot) \in L_{2}(T ; U): u(t) \in P \text { for a. a. } t \in T\right\},
$$

$P \subset U$ is a convex bounded and closed set.

The problem under consideration consists in the following. Let Eqn. (1), an action $f(\cdot)$, an initial state $x_{0}$, and the set of admissible inputs $U_{*}$ be known. In addition, at moments $t \in T$, values

$$
z(t)=G x(t)
$$

are inaccurately measured. Here $G$ is a given linear continuous operator acting from the space of states $H$ into a Hilbert space of measurements $H_{1}$. Results of the measurements $\xi^{h}(t)$ are, generally speaking, inaccurate:

$$
\left|\xi^{h}(t)-z(t)\right|_{H_{1}} \leq h, \quad t \in T .
$$

Here $h$ is the measurement accuracy.

Let $U_{z}$ be the set of all admissible inputs $u(\cdot)$ compatible with some output $z(\cdot)$, i.e.,

$$
\begin{gathered}
U_{z}=\left\{u(\cdot) \in U_{*}: G x\left(t ; 0, x_{0}, u(\cdot)\right)=z(t), \quad \forall t \in T\right\}, \\
J(u(\cdot))=\int_{0}^{\vartheta} \omega(t, u(t)) \mathrm{d} t
\end{gathered}
$$

is a given performance index. Here $\omega(\cdot, \cdot): T \times U \rightarrow$ $\mathbb{R}_{+}=[0,+\infty)$ is a functional, which is convex with respect to the second argument. It is assumed that the functional $J(u(\cdot))$ is defined on the set $U_{*}$ and is lower semicontinuous. It is necessary to construct a stable algorithm for calculating an extremal value

$$
J_{z}^{0}=\min \left\{J(u(\cdot)): u(\cdot) \in U_{z}\right\}
$$

and an extremal input action $u_{0}(\cdot) \in U_{0}(z)$, where

$$
U_{0}(z)=\arg \min \left\{J(u(\cdot)): u(\cdot) \in U_{z}\right\} .
$$

Since the functional $J$ is convex and the set $U_{*}$ is bounded and closed, the set $U_{0}(z)$ is a non-empty convex and closed set. Therefore, the problem in question has a solution. However, the precise calculation of $J_{z}^{0}$ and $u_{0}(\cdot)$ is impossible, in particular, due to inaccuracies in measuring the values $z(t), t \in T$ (see (3)). In this case, it is necessary to design a stable algorithm for approximate determination of the value $J_{z}^{0}$ and the extremal input action $u^{h}(\cdot)=u\left(\cdot ; \xi^{h}(\cdot)\right)$. The stability of the algorithm is understood in the following sense:

$$
\begin{aligned}
J\left(u^{h}(\cdot)\right) \rightarrow J_{z}^{0}, & u^{h}(\cdot) \rightarrow U_{0}(z) \\
& \text { weakly in } L_{2}(T ; U) \text { as } h \rightarrow+0 .
\end{aligned}
$$


The last relation means that any convergent (in $L_{2}(T ; U)$ ) sequence $\left\{u^{h_{l}}(\cdot)\right\}, h_{l} \rightarrow+0$ as $l \rightarrow+\infty$, converges to some element from the set $U_{0}(z)$. Below we present an algorithm for solving the problem considered in the case when the operators $A$ and $B$, as well as the functional $J$ and the initial state $x_{0}$, are inaccurately known.

Note that the problem of source function reconstruction formulated above can be interpreted as an optimal control problem for the parabolic equation (1) subject to state constraints. Then the performance index takes the form (4), whereas the state constraints are given by (2). A solving algorithm suggested in the paper is oriented to the case of inaccurate data on the problem parameters, i.e., on the system structure, the quality criterion, and the state constraints.

\section{Auxiliary results}

Before passing on to solving the problem in question, let us give auxiliary statements. Introduce the operator $F: L_{2}(T ; U) \rightarrow L_{2}\left(T ; H_{1}\right)$ and the element $b(\cdot) \in$ $L_{2}\left(T ; H_{1}\right)$ :

$$
\begin{aligned}
(F u(\cdot))(\eta) & =\int_{0}^{\eta} G S(\eta-t) B u(t) \mathrm{d} t, \quad \eta \in T, u(\cdot) \in U_{*}, \\
b(\eta) & =z(\eta)-G S(\eta) x_{0}, \quad \eta \in T .
\end{aligned}
$$

Here the function $z$ is defined by (2). Then the problem of calculating the extremal value $J_{z}^{0}$ and extremal input action $u_{0}(\cdot) \in U_{0}(z)$ is equivalent to the following extremal problem.

It is required to find

$$
u^{0}=\arg \min \left\{J(u): u \in U_{*}, F u=b\right\}
$$

and

$$
J^{0}=\min \left\{J(u): u \in U_{*}, F u=b\right\},
$$

where $J$ is the quality criterion (4). Note that the set $\{u \in$ $\left.U_{*}: F u=b\right\}$ is non-empty. Therefore, there exists a solution to the last problem, and $J^{0}=J_{z}^{0}$ (see (5)). If the function $\omega(\cdot, \cdot)$ is strictly convex with respect to the second argument, then the set $U_{0}(z)$ is a singleton and, in addition, $u^{0}=u_{0}=U_{0}(z)$.

Let non-negative numbers $\nu^{F}, \nu^{b}$, and $\nu^{J}$, linear continuous operators $F^{\nu}: L_{2}(T ; U) \rightarrow L_{2}\left(T ; H_{1}\right)$, elements $b^{\nu} \in L_{2}\left(T ; H_{1}\right)$, and convex functionals $J^{\nu}(\cdot)$ defined on $U_{*}$ be given in such a way that

$$
\begin{aligned}
\left|F^{\nu} u-F u\right|_{L_{2}\left(T ; H_{1}\right)} & \leq \nu^{F}, \quad \forall u \in U_{*}, \\
\left|b^{\nu}-b\right|_{L_{2}\left(T ; H_{1}\right)} \leq \nu^{b}, & \\
\left|J^{\nu}(u)-J(u)\right| & \leq \nu^{J}, \quad \forall u \in U_{*} .
\end{aligned}
$$

For simplicity, in what follows it is assumed that $\nu^{F}, \nu^{b}, \nu^{J} \in[0,1)$.

Our goal is to design an algorithm for approximate determination of the value $J^{0}$ and the element $u^{0}$. Namely, it is required to construct an algorithm which, using values $F^{\nu}, b^{\nu}$, and $J^{\nu}$ known instead of $F, b$, and $J$, forms elements $\left\{u_{j}\right\} \in U_{*}, j=1, \ldots$, with the properties

$$
\begin{gathered}
\left|F u_{j}-b\right|_{L_{2}\left(T ; H_{1}\right)} \rightarrow 0, \\
J\left(u_{j}\right) \rightarrow J^{0} \quad \text { as } \quad j \rightarrow+\infty,
\end{gathered}
$$

if $\nu^{F}=\nu_{j}^{F} \rightarrow 0, \nu^{b}=\nu_{j}^{b} \rightarrow 0$, and $\nu^{J}=\nu_{j}^{J} \rightarrow 0$ as $j \rightarrow+\infty$.

Let us pass on to the description of this algorithm.

Set

$$
\begin{aligned}
K_{0} & =\sup \left\{J(u): u \in U_{*}\right\}, \\
U_{\gamma} & =\left\{u \in U_{*}:|F u-b|_{L_{2}\left(T ; H_{1}\right)} \leq \gamma\right\}, \\
J^{0}(\gamma) & =\inf \left\{J(u): u \in U_{\gamma}\right\} .
\end{aligned}
$$

From the results of (Vasiliev, 1981, p. 182), we obtain what follows.

Theorem 1. Let $u_{j} \in U_{*}, \alpha_{j}>0, \gamma_{j}>0, \mid J^{\nu_{j}}(u)-$ $J(u) \mid \leq \nu_{j}^{J} \forall u \in U_{*}$,

$$
\begin{gathered}
\left|F u_{j}-b\right|_{L_{2}\left(T ; H_{1}\right)}^{2}+\alpha_{j} J\left(u_{j}\right)-\alpha_{j} J^{0} \leq \gamma_{j}, \\
\nu_{j}^{J} \rightarrow 0, \quad \alpha_{j} \rightarrow+0, \quad \gamma_{j} \rightarrow+0, \\
\gamma_{j} / \alpha_{j} \rightarrow 0 \quad \text { as } \quad j \rightarrow+\infty .
\end{gathered}
$$

Then we have

(a) $J^{\nu_{j}}\left(u_{j}\right) \rightarrow J^{0}$ as $j \rightarrow+\infty$,

(b)

$$
\begin{aligned}
& J^{0}\left(\left(\gamma_{j}+2 K_{0} \alpha_{j}\right)^{1 / 2}\right)-\nu_{j}^{J} \\
& \leq J^{\nu_{j}}\left(u_{j}\right) \leq J^{0}+\gamma_{j} / \alpha_{j}+\nu_{j}^{J} .
\end{aligned}
$$

Any element $w_{*} \in W$ is called an $\varepsilon$-solution $(\varepsilon>0)$ of the extremal problem $\varphi(w) \rightarrow$ inf, $w \in W \neq \varnothing$, if $\varphi\left(w_{*}\right) \leq \inf \{\varphi(w): w \in W\}+\varepsilon$. We denote by the symbol $Y_{j}^{\nu}(\delta, \alpha, \varepsilon)$ the set of elements $y_{i}, i=0,1, \ldots, j$, from $U$ such that

$$
\begin{aligned}
y_{0} & =0, \\
y_{i+1} & =y_{i}+u_{i} \delta, \quad i=0,1, \ldots, j-1,
\end{aligned}
$$

where $u_{i}$ is an $\varepsilon$-solution of the problem

$$
2\left\langle F^{\nu} y_{i}-i \delta b^{\nu}, F^{\nu} u\right\rangle+\alpha J^{\nu}(u) \rightarrow \inf , \quad u \in U_{*} .
$$

Hereinafter, the symbol $\langle\cdot, \cdot\rangle$ denotes the scalar product in $L_{2}\left(T ; H_{1}\right)$.

Let

$$
K_{1}=|b|_{L_{2}\left(T ; H_{1}\right)}, \quad|F u|_{L_{2}\left(T ; H_{1}\right)} \leq K_{2} \forall u \in U_{*} .
$$

Based on the approach from (Kryazhimskii and Osipov, 1987; Kryazhimskii et al., 1997), the following lemma is proved. 


\section{Lemma 1. The inequality}

$$
\begin{aligned}
\left|F\left(y_{j} /(\delta j)\right)-b\right|_{L_{2}\left(T ; H_{1}\right)}^{2} & +\alpha\left\{J\left(y_{j} /(\delta j)\right)-J^{0}\right\} /(\delta j) \leq \delta_{j}^{*}
\end{aligned}
$$

is valid for any $j \geq 1$, where

$$
\begin{aligned}
\delta_{j}^{*}= & k_{1} \nu^{F}+k_{2} \nu^{b}+\left(k_{3}\left(\nu^{F}\right)^{2}+2 \alpha \nu^{J}\right) /(j \delta) \\
& +k_{4} / j+\varepsilon /(\delta j), \\
k_{1}= & 4\left(K_{1}+2 K_{2}\right), \\
k_{2}= & K_{2}+1, \\
k_{3}= & 4, \\
k_{4}= & \left(K_{1}+K_{2}\right)^{2} .
\end{aligned}
$$

Proof. Let us estimate the change of the value

$$
\begin{aligned}
\Lambda_{i+1}= & \left|F\left(y_{i}+\delta u_{i}\right)-t_{i+1} b\right|_{L_{2}\left(T ; H_{1}\right)}^{2} \\
& +\alpha \int_{0}^{t_{i+1}} J(\dot{y}(\tau)) \mathrm{d} \tau-\alpha J\left(u^{0}\right) t_{i+1} \\
= & \Lambda_{i}+\mu_{i}+\delta^{2}\left|F u_{i}-b\right|_{L_{2}\left(T ; H_{1}\right)}^{2}, \quad i \geq 0,
\end{aligned}
$$

where

$$
\begin{aligned}
& \dot{y}(t)=u_{i} \quad \text { for } \quad t \in\left[t_{i}, t_{i+1}\right), \\
& i=0,1, \ldots, \quad t_{i}=i \delta, \quad y(0)=0, \\
& \mu_{i}=2\left\langle F y_{i}-t_{i} b, F u_{i}-b\right\rangle \delta+\alpha \delta\left\{J\left(u_{i}\right)-J\left(u^{0}\right)\right\} .
\end{aligned}
$$

Since $u^{0}$ is a solution of the problem under consideration, the equality

$$
F u^{0}=b
$$

is fulfilled. Therefore,

$$
\begin{aligned}
\mu_{i}= & 2\left\langle F y_{i}-t_{i} b, F u_{i}-b\right\rangle \delta-2\left\langle F y_{i}-t_{i} b, F u^{0}-b\right\rangle \delta \\
& +\alpha \delta\left\{J\left(u_{i}\right)-J\left(u^{0}\right)\right\} \\
= & 2\left\langle F y_{i}-t_{i} b, F u_{i}\right\rangle \delta+\alpha \delta J\left(u_{i}\right) \\
& \quad-2\left\langle F y_{i}-t_{i} b, F u^{0}\right\rangle \delta-\alpha \delta J\left(u^{0}\right) .
\end{aligned}
$$

Moreover,

$$
\begin{gathered}
\lambda_{i}^{*} \equiv 2\left\langle F y_{i}-t_{i} b, F u_{i}\right\rangle \delta+\alpha J\left(u_{i}\right) \delta \\
-2\left\langle F^{\nu} y_{i}-t_{i} b^{\nu}, F^{\nu} u_{i}\right\rangle \delta+\alpha J^{\nu}\left(u_{i}\right) \delta=\sum_{j=1}^{3} \lambda^{(j)}, \\
\lambda_{i}^{(1)}=2\left\langle F y_{i}-t_{i} b,\left(F-F^{\nu}\right) u_{i}\right\rangle \delta, \\
\lambda_{i}^{(2)}=2\left\langle\left(F-F^{\nu}\right) y_{i}-t_{i}\left(b-b^{\nu}\right), F^{\nu} u_{i}\right\rangle \delta, \\
\lambda_{i}^{(3)}=\alpha\left\{J\left(u_{i}\right)-J^{\nu}\left(u_{i}\right)\right\} \delta .
\end{gathered}
$$

In addition, by we obtain

$$
\begin{array}{r}
\left|F y_{i}\right|_{L_{2}\left(T ; H_{1}\right)}=\left|\sum_{j=0}^{i-1} F u_{j} \delta\right|_{L_{2}\left(T ; H_{1}\right)} \leq t_{i-1} K_{2}, \\
\left|\left(F-F^{\nu}\right) y_{i}\right|_{L_{2}\left(T ; H_{1}\right)} \\
=\left|\sum_{j=0}^{i-1}\left(F-F^{\nu}\right) u_{j} \delta\right|_{L_{2}\left(T ; H_{1}\right)} \leq \nu^{F} t_{i},
\end{array}
$$

$$
\begin{aligned}
\left|F^{\nu} u\right|_{L_{2}\left(T ; H_{1}\right)} & \leq\left|\left(F^{\nu}-F\right) u\right|_{L_{2}\left(T ; H_{1}\right)}+|F u|_{L_{2}\left(T ; H_{1}\right)} \\
& \leq K_{2}+\nu^{F}, \quad u \in U_{*} .
\end{aligned}
$$

Note that, due to the convexity and completeness of the set $P$, the rule for choosing the elements $u_{i}$ implies the inclusion $y_{i} /(\delta i) \in U_{*}$. Thus, we have (see (6)-(8))

$$
\begin{aligned}
& \lambda_{i}^{(1)} \leq 2\left(K_{2}+K_{1}\right) \nu^{F} \delta t_{i}, \\
& \lambda_{i}^{(2)}=2\left(\nu^{F}+\nu^{b}\right)\left(K_{2}+\nu^{F}\right) \delta t_{i}, \\
& \lambda_{i}^{(3)} \leq \alpha \delta \nu^{F} .
\end{aligned}
$$

Therefore,

$$
\lambda_{i}^{*} \leq \delta \nu^{F} K_{1 i}+\delta \nu^{b} K_{2 i}+\alpha \nu^{J} \delta,
$$

where

$$
\begin{aligned}
& K_{1 i}=2\left(\left(2 K_{2}+K_{1}\right) t_{i}+\nu^{F}\right), \\
& K_{2 i}=2 t_{i}\left(K_{2}+\nu^{F}\right) \delta .
\end{aligned}
$$

A similar estimate holds if we replace $u_{i}$ by $u^{0}$. Hence,

$$
\begin{aligned}
\mu_{i} \leq & 2\left\langle F^{\nu} y_{i}-t_{i} b^{\nu}, F^{\nu} u_{i}\right\rangle \delta-2\left\langle F^{\nu} y_{i}-t_{i} b^{\nu}, F^{\nu} u^{0}\right\rangle \delta \\
& +\alpha \delta\left\{J^{\nu}\left(u_{i}\right)-J^{\nu}\left(u^{0}\right)\right\} \\
& +2\left\{K_{1 i} \nu^{F}+K_{2 i} \nu^{b}+\alpha \nu^{J}\right\} \delta .
\end{aligned}
$$

From (14) we deduce that

$$
\mu_{i} \leq 2\left\{K_{1 i} \nu^{F}+K_{2 i} \nu^{b}+\alpha \nu^{J}\right\} \delta+\varepsilon \delta .
$$

It is easily seen that

$$
\left|F u_{i}-b\right|_{L_{2}\left(T ; H_{1}\right)}^{2} \delta^{2} \leq\left\{\left(K_{2}+K_{1}\right) \delta\right\}^{2} .
$$

In addition, due to the convexity of $J$, we obtain

$$
J(y(t) / t)=J\left(\frac{1}{t} \int_{0}^{t} \dot{y}(\tau) \mathrm{d} \tau\right) \leq \frac{1}{t} \int_{0}^{t} J(\dot{y}(\tau)) \mathrm{d} \tau .
$$

Accordingly,

$$
\begin{aligned}
\Lambda_{i+1} \leq & \Lambda_{i}+2\left\{K_{1 i} \nu^{F}+K_{2 i} \nu^{b}+\alpha \nu^{J}\right\} \delta \\
& +\left\{\left(K_{2}+K_{1}\right) \delta\right\}^{2}+\varepsilon \delta \\
\leq & \Lambda_{i}+2\left\{2 \left(\left(2 K_{2}+K_{1}\right)\left(i \delta+\nu^{F}\right) \nu^{F} \delta\right.\right. \\
& \left.+2 i \delta\left(K_{2}+\nu^{F}\right) \nu^{b} \delta+\alpha \nu^{J} \delta\right\} \\
& +\left\{\left(K_{2}+K_{1}\right) \delta\right\}^{2}+\varepsilon \delta, \quad i \geq 0 .
\end{aligned}
$$

Using (12), we obtain $\Lambda_{0}=0$. Then

$$
\begin{aligned}
\Lambda_{i+1} \leq & 2\left\{\left(\left(2 K_{1}+4 K_{2}\right) i \delta+2 \nu^{F}\right) \nu^{F} \delta\right. \\
& \left.+2 i \delta\left(K_{2}+\nu^{F}\right) \nu^{b} \delta+\alpha \nu^{J} \delta\right\} i \\
& +\left\{\left(K_{1}+K_{2}\right) \delta\right\}^{2} i+\varepsilon \delta i, \quad i \geq 0 .
\end{aligned}
$$


Dividing the right-hand and left-hand sides by $t_{i+1}^{2}$ and using (15), we get

$$
\begin{aligned}
\mid F & \left(y\left(t_{i+1}\right) / t_{i+1}\right)-\left.b\right|_{L_{2}\left(T ; H_{1}\right)} ^{2} \\
& +\alpha / t_{i+1}\left\{J\left(y\left(t_{i+1}\right) / t_{i+1}\right)-J\left(u^{0}\right)\right\} \\
\leq & 2\left\{\frac{2 K_{1}+4 K_{2}}{\delta}+\frac{2 \nu^{F}}{(i+1) \delta^{2}}\right\} \nu^{F} \delta+\varepsilon /(\delta(i+1)) \\
& +4\left(K_{2}+\nu^{F}\right) \nu^{b}+2 \alpha \nu^{J} /((i+1) \delta) \\
& +\left(K_{1}+K_{2}\right)^{2} /(i+1) \\
= & 4\left\{\left(K_{1}+2 K_{2}\right) \nu^{F}+\left(K_{2}+\nu^{F}\right) \nu^{b}\right\} \\
& +2\left\{2\left(\nu^{F}\right)^{2}+\alpha \nu^{J}\right\} /((i+1) \delta) \\
& +\left(K_{1}+K_{2}\right)^{2} /(i+1)+\varepsilon /(\delta(i+1)) .
\end{aligned}
$$

The next theorem follows from Theorem 1 and Lemma 1.

\section{Theorem 2. Let}

(a) $\delta_{j} \rightarrow+0$, $\varepsilon_{j} \rightarrow+0$ as $j \rightarrow+\infty$;

(b) sequences $\left\{\alpha_{j}\right\}$ and $\left\{\gamma_{j}\right\}$ satisfy the conditions (11);

(c) inequalities

$$
\begin{aligned}
k_{1} \nu_{j}^{F}+k_{2} \nu_{j}^{b}+\left(k_{3}\left(\nu_{j}^{F}\right)^{2}+2 \alpha_{j} \nu_{j}^{J}\right) /\left(j \delta_{j}\right) \\
+k_{4} / j+\varepsilon_{j} /\left(\delta_{j} j\right) \leq \gamma_{j}
\end{aligned}
$$

be true;

(d) $\left\{y_{i}\right\}_{i=0}^{j}=Y_{j}^{\nu}\left(\delta_{j}, \alpha_{j}, \varepsilon_{j}\right)$.

Then the sequence of elements $\left\{u_{j}\right\}_{j=1}^{+\infty}$,

$$
u_{j}=y_{j} /\left(\delta_{j} j\right) \in U_{*},
$$

satisfying the conditions (9) and (10), solves the problem of approximate calculation of $u^{0}$ and $J^{0}$.

As is seen from Lemma 1 and Theorem 2, choosing appropriate relations between the values $\delta, i, \nu^{F}, \nu^{b}, \nu^{J}$, and $\alpha$, we provide a "slow" growth of the Lyapunov functional

$$
\begin{aligned}
\Lambda(t)=|F(y(t) / t)-b|_{L_{2}\left(\cdot ; H_{1}\right)}^{2} & \\
& +\alpha \int_{0}^{t} J(\dot{y}(\tau)) \mathrm{d} \tau / t-\alpha J\left(u^{0}\right) / t, t>0 .
\end{aligned}
$$

The rule (14) for choosing controls $u_{i}$ is, in essence, a modification of the extremal shift principle from the theory of differential games (Krasovskii and Subbotin, 1988).

Let

$$
\begin{aligned}
& \nu_{1}\left(\alpha, \delta, j, \varepsilon, \nu^{F}, \nu^{b}, \nu^{J}\right)=\delta_{j}^{*}+2 K_{0} \alpha /(\delta j), \\
& \nu_{2}\left(\alpha, \delta, j, \varepsilon, \nu^{F}, \nu^{b}, \nu^{J}\right)=\delta_{j}^{*} \delta j / \alpha .
\end{aligned}
$$

The next inequalities follow from Lemma 1:

$$
\begin{gathered}
\left|F\left(y_{j} /(\delta j)\right)-b\right|_{L_{2}\left(T ; H_{1}\right)}^{2} \leq \nu_{1}\left(\alpha, \delta, j, \varepsilon, \nu^{F}, \nu^{b}, \nu^{J}\right), \\
J\left(y_{j} /(\delta j)\right)-J^{0} \leq \nu_{2}\left(\alpha, \delta, j, \varepsilon, \nu^{F}, \nu^{b}, \nu^{J}\right) .
\end{gathered}
$$

The following lemma holds.

Lemma 2. Let $\nu_{j}^{F} \rightarrow 0, \nu_{j}^{b} \rightarrow 0, \nu_{j}^{J} \rightarrow 0, \alpha_{j} \rightarrow+0$, $\alpha_{j} /\left(\delta_{j} j\right) \rightarrow+0,\left(\delta_{j}+\varepsilon_{j}\right) / \alpha_{j} \rightarrow+0, \varepsilon_{j} /\left(\delta_{j} j\right) \rightarrow+0$, $\nu_{j}^{F} j \delta_{j} / \alpha_{j} \rightarrow 0, \nu_{j}^{b} j \delta_{j} / \alpha_{j} \rightarrow 0$ as $j \rightarrow+\infty$.

Then

$$
\begin{aligned}
\left|F\left(y_{j} /\left(\delta_{j} j\right)\right)-b\right|_{L_{2}\left(T ; H_{1}\right)}^{2} & \rightarrow 0 \\
J\left(y_{j} /\left(\delta_{j} j\right)\right) & \rightarrow J^{0} \text { as } j \rightarrow+\infty .
\end{aligned}
$$

\section{Condition 1.}

$$
\begin{gathered}
j=j(h)=[1 / h], \quad \nu_{j}^{F}=a_{1} h, \quad \nu_{j}^{b}=a_{2} h, \\
\nu_{j}^{J}=a_{3} h, \quad \varepsilon_{j}=a_{4} \delta_{j}, \quad \alpha_{j}=\delta_{j}^{1 / 2}, \\
\delta_{j}=h^{1-\varkappa}, \quad \varkappa=\mathrm{const} \in(0,1) .
\end{gathered}
$$

Here the symbol $[1 / h]$ denotes the integer part of $1 / h ; a_{j}, j=1, \ldots, 4$, are some constants. In this case, $j=j(h) \rightarrow+\infty$ as $h \rightarrow+0$ and the elements $y_{j} /\left(\delta_{j} j\right)$ depend on $h$, i.e.,

$$
u^{h}=y_{j(h)} /\left(h^{1-\varkappa}[1 / h]\right) \in U_{*} .
$$

The next statement can be formulated.

Corollary 1. Let Condition 1 be fulfilled and $h \in$ $\left(0,1-\varepsilon_{*}\right), \varepsilon_{*}=$ const $\in(0,1)$. Then the inequalities

$$
\begin{aligned}
\left|F\left(u^{h}\right)-b\right|_{L_{2}\left(T ; H_{1}\right)}^{2} & \leq C h, \\
J\left(u^{h}\right)-J^{0} & \leq C_{0} h^{1 / 2-\varkappa / 2}
\end{aligned}
$$

hold. Here $C=C\left(\varepsilon_{*}\right), C_{0}=C_{0}\left(\varepsilon_{*}\right)$, and the element $u^{h}$ is defined by (18).

The validity of Corollary 1 follows from the inequalities

$$
\begin{aligned}
& \nu_{1}\left(\alpha, \delta, j, \varepsilon, \nu^{F}, \nu^{b}, \nu^{J}\right) \leq C h, \\
& \nu_{2}\left(\alpha, \delta, j, \varepsilon, \nu^{F}, \nu^{b}, \nu^{J}\right) \leq C_{0} h^{1 / 2-\varkappa / 2}
\end{aligned}
$$

and the inequalities (16) and (17).

Let a sequence of positive numbers $\left\{h_{l}\right\}_{l=0}^{+\infty}, h_{l} \rightarrow$ +0 as $l \rightarrow+\infty$ be fixed. Let Condition 1 be fulfilled. The symbol $y_{l}$ is used for the sequence constructed according to (12)-114) for $j=j_{l}=\left[1 / h_{l}\right], h=h_{l}, \delta=\delta_{l}=h_{l}^{1-\varkappa}$, and $\varkappa=$ const $\in[0,1)$. Then the following theorem is valid.

Theorem 3. Let $u_{l}=y_{j_{l}} /\left(j_{l} h_{l}^{1-\varkappa}\right), l=1,2, \ldots$. Then any weakly convergent subsequence of the sequence $\left\{u_{l}\right\}_{l=1}^{+\infty}$ weakly converges in $L_{2}(T ; U)$ to the set $U_{0}(z)$. If $J(u)=|u|_{L_{2}(T ; U)}^{2}$, then this convergence is strong. 


\section{Solving the algorithm}

Let us come back to the problem of approximate calculating of the value $J_{z}^{0}$ and the extremal input action $u^{h}(\cdot)$. Let the operators $A$ and $B$, as well as the initial state $x_{0}$, be inaccurately known. Namely, we have some operators $A^{\nu}: V \rightarrow V$ and $B^{\nu}: U \rightarrow H$, and also an element $x_{0}^{\nu} \in H$ such that

$$
\begin{aligned}
\left|B^{\nu}-B\right|_{L(U ; H)} & \leq \nu^{B} \\
\left|x_{0}^{\nu}-x_{0}\right|_{H} & \leq \nu^{x_{0}} .
\end{aligned}
$$

The operator $A^{\nu}$ generates a semigroup of linear continuous operators $S^{\nu}(t), t \geq 0$, such that

$$
\sup _{t \in T}\left|S^{\nu}(t)-S(t)\right|_{L(H ; H)} \leq \nu^{A} .
$$

We also assume that, instead of the functional $\omega(t, u)$, we get functionals $\omega^{\nu}(t, u)$ which are convex with respect to $u$, such that the functionals

$$
J^{\nu}(u(\cdot))=\int_{0}^{\vartheta} \omega^{\nu}(t, u(t)) \mathrm{d} t,
$$

are defined on the set $U_{*}$, are lower semicontinuous and satisfy the inequalities

$$
\left|J^{\nu}(u(\cdot))-J(u(\cdot))\right| \leq \nu^{I}, \quad \forall u(\cdot) \in U_{*} .
$$

Here $\nu^{A}, \nu^{B}, \nu^{x_{0}}$, and $\nu^{I} \in[0,1)$ are given numbers.

Let the sequence $\left\{\left(y_{i}(\cdot), \psi_{i}(\cdot)\right\}_{i=0}^{j}\right.$ of elements from $L_{2}(T ; U) \times L_{2}(T ; H)$ be defined by the rule:

$$
\begin{aligned}
y_{i+1}(\cdot) & =y_{i}(\cdot)+\delta w_{i}(\cdot) \in L_{2}(T ; U), \quad y_{0}(\cdot)=0 \\
\psi_{i+1}(\cdot) & =\psi_{i}(\cdot)+\delta \zeta_{i}(\cdot) \in L_{2}(T ; H), \\
\psi_{0}(\cdot) & =0, \quad i=0,1, \ldots, j-1,
\end{aligned}
$$

where $w_{i}(\cdot)$ is a $\beta_{0}$-solution to the problem

$$
\begin{gathered}
\int_{0}^{\vartheta}\left\{2\left(B^{\nu *} \psi_{i}(t), w(t)\right)_{U}+\alpha \omega^{\nu}(t, w(t))\right\} \mathrm{d} t \rightarrow \inf , \\
w(\cdot) \in U_{*} \\
\gamma_{i}(\cdot) \in C(T, H), \quad\left|\gamma_{i}(\cdot)-\bar{\gamma}_{i}(\cdot)\right|_{C(T, H)} \leq \beta_{2}
\end{gathered}
$$

$\bar{\gamma}_{i}(\cdot)$ is a solution on $T$ to the Cauchy problem

$$
\begin{gathered}
\dot{\gamma}(t)=A^{\nu} \gamma(t)+B^{\nu} w_{i}(t), \quad \gamma(0)=0 \\
\left|\zeta_{i}(\cdot)-\bar{\zeta}_{i}(\cdot)\right|_{C(T, H)} \leq \beta_{1}
\end{gathered}
$$

$\bar{\zeta}_{i}(\cdot)$ is a solution on $T$ to the Cauchy problem

$$
\begin{aligned}
\dot{\zeta}(t) & =-A^{\nu *} \zeta(t)-G^{*} \kappa_{i}(t), \quad \zeta(\vartheta)=0 ; \\
\kappa_{i}(t) & =G \gamma_{i}(t)-b^{\nu}(t), \quad t \in T .
\end{aligned}
$$

Here the symbol $G^{*}$ stands for the operator adjoint to $G$,

$$
b^{\nu}(t)=\xi^{h}(t)-G S^{\nu}(t) x_{0}^{\nu} .
$$

Note that, in this case, we have

$$
\begin{aligned}
& \left(F^{\nu} u(\cdot)\right)(\eta) \\
& \quad=\int_{0}^{\eta} G S^{\nu}(\eta-t) B^{\nu} u(t) \mathrm{d} t, \quad \eta \in T, u(\cdot) \in U_{*} .
\end{aligned}
$$

Let

$$
\begin{aligned}
\psi_{i+1}^{*}(\cdot)=\psi_{i}^{*}(\cdot)+\delta \zeta_{i}^{*}(\cdot) \in & L_{2}(T ; H), \\
& i=0,1, \ldots, j-1,
\end{aligned}
$$

where $\zeta_{i}^{*}(\cdot)$ is a solution on $T$ of the problem

$$
\begin{aligned}
\dot{\varrho}(t) & =-A^{\nu *} \varrho(t)-G^{*} \kappa_{i}^{*}(t), & \varrho(\vartheta) & =0, \\
\kappa_{i}^{*}(t) & =G \bar{\gamma}_{i}(t)-b^{\nu}(t), & t & \in T .
\end{aligned}
$$

Here $\bar{\gamma}_{i}(\cdot)$ is a solution of the problem (28) on $T$.

Lemma 3. For any $w(\cdot) \in U_{*}$, the following equality

$$
\begin{gathered}
\int_{0}^{\vartheta}\left\{2\left(B^{\nu *} \psi_{i}^{*}(t), w(t)\right)_{U}+\alpha \omega^{\nu}(t, w(t))\right\} \mathrm{d} t \\
=2 \Psi_{i}(w(\cdot))+\alpha J^{\nu}(w(\cdot)), \\
i=0, \ldots, j-1,
\end{gathered}
$$

holds, where

$$
\Psi_{i}(w(\cdot))=\left\langle F^{\nu} y_{i}(\cdot)-i \delta b^{\nu}, F^{\nu} w(\cdot)\right\rangle .
$$

Proof. Taking into account the structure of the functional $J^{\nu}(\cdot)$ (23), we conclude that (35) is equivalent to the equality

$$
\begin{gathered}
\int_{0}^{\vartheta}\left(B^{\nu *} \psi_{i}^{*}(t), w(t)\right)_{U} \mathrm{~d} t=\Psi_{i}(w(\cdot)), \\
i=0, \ldots, j-1 .
\end{gathered}
$$

Introduce the notation

$$
\begin{aligned}
\nu_{i}(\eta) & =\left(F^{\nu} y_{i}(\cdot)\right)(\eta)-i \delta b^{\nu}(\eta) \\
& =\int_{0}^{\eta} G S^{\nu}(\eta-t) B^{\nu} y_{i}(t) \mathrm{d} t-i \delta b^{\nu}(\eta), \quad \eta \in T .
\end{aligned}
$$

Then

$$
\Psi_{i}(w(\cdot))=\int_{0}^{\vartheta}\left(\nu_{i}(\eta) \mathrm{d} \eta,\right.
$$




$$
\begin{aligned}
\left.\int_{t_{0}}^{\eta} G S^{\nu}(\eta-t) B^{\nu} w(t) \mathrm{d} t\right)_{H_{1}} \mathrm{~d} \eta \\
=\int_{0}^{\vartheta}\left(B^{\nu *} \chi_{i}(t), w(t)\right)_{U} \mathrm{~d} t,
\end{aligned}
$$

where the symbol $(\cdot, \cdot)_{H_{1}}$ stands for the scalar product in $H_{1}$,

$$
\chi_{i}(t)=\int_{t}^{\vartheta} S^{\nu *}(\eta-t) G^{*} \nu_{i}(\eta) \mathrm{d} \eta .
$$

To prove (35), it is sufficient to show that

$$
\psi_{i}^{*}(\cdot)=\chi_{i}(\cdot)
$$

for $i=0, \ldots, j-1$.

Let us prove (40) by induction. For $i=0$, we have $y_{0}(\cdot)=0$. By virtue of (38) and (39), we obtain $\chi_{0}(\cdot)=$ $0=\psi_{0}^{*}(\cdot)$. Assume that the equalities (40) hold for some $i<j-1$. Show that the equality

$$
\psi_{i+1}^{*}(\cdot)=\chi_{i+1}(\cdot)
$$

is also true. From (38) and (24), it follows that

$$
\nu_{i+1}(\eta)=\nu_{i}(\eta)+\delta\left\{G \phi_{i}(\eta)-b^{\nu}(\eta)\right\},
$$

where

$$
\phi_{i}(\eta)=\int_{0}^{\eta} S^{\nu}(\eta-t) B^{\nu} w_{i}(t) \mathrm{d} t .
$$

Note that $\phi_{i}(\cdot)$ is a solution of the Cauchy problem (28), i. e., $\phi_{i}(\cdot)=\bar{\gamma}_{i}(\cdot)$. Then (see (42) and (33))

$$
\nu_{i+1}(\cdot)=\nu_{i}(\cdot)+\delta \kappa_{i}^{*}(\cdot) .
$$

Further, from (39) and (43), it follows that

$$
\chi_{i+1}(\cdot)=\chi_{i}(\cdot)+\delta \rho_{i}(\cdot) .
$$

where

$$
\rho_{i}(t)=\int_{t}^{\vartheta} S^{\nu *}(\eta-t) G^{*} \kappa_{i}^{*}(\eta) \mathrm{d} \eta .
$$

Consequently, the function $\rho_{i}(\cdot)$ is a solution of the Cauchy problem $(33)$, i.e., $\rho_{i}(\cdot)=\bar{\zeta}_{i}(\cdot)$. Hence, due to (44), (34), and (40), we get (41).

Introduce the constants

$$
\begin{aligned}
C_{1} & =\sup \left\{|u|_{U}: u \in U_{*}\right\}, \\
C_{2} & =\left(|B|_{L(U ; H)}+1\right) C_{1} \vartheta^{1 / 2}, \\
C_{3} & =|G|_{L\left(H ; H_{1}\right)}^{2} \vartheta K_{3}, \\
K_{3} & =\sup _{t \in T} \sup _{\nu \in(0,1]}\left|S^{\nu}(t)\right|_{L(H ; H)} .
\end{aligned}
$$

Lemma 4. Let the elements $\left\{y_{i}(\cdot), \psi_{i}(\cdot)\right\}$ be defined according to (24)-(31). Then $\left\{y_{i}(\cdot)\right\}_{i=0}^{j}=Y_{j}^{\nu}(\delta, \alpha, \varepsilon)$, where

$$
\varepsilon=j C_{2} \delta\left(\beta_{1}+C_{3} \beta_{2}\right)+\beta_{0} .
$$

Proof. By definition, $w_{i}(\cdot)$ is a $\beta_{0}$-solution of the problem (26). Taking into account (35), we show that, for all $\beta_{0}, \beta_{1}, \beta_{2} \geq 0, w_{i}(\cdot)$ is an $\varepsilon$-solution of the problem

$$
2 \Psi_{i}(w(\cdot))+\alpha J^{\nu}(w(\cdot)) \rightarrow \inf , \quad w(\cdot) \in U_{*} .
$$

It is sufficient to prove that $w_{i}(\cdot)$ is an $\varepsilon_{i}$-solution of the problem (45), where $\varepsilon_{i}=\mu_{i}+\beta_{0}$,

$$
\mu_{i}=i C_{2} \delta\left(\beta_{1}+C_{3} \beta_{2}\right)
$$

(clearly, $\varepsilon_{i} \leq \varepsilon$ for $i \leq j$ ). For this purpose, it is sufficient to prove that the values of the functionals to be minimized in the problems (26) and (45) (for an arbitrary $w(\cdot) \in U_{*}$ ) differ by no more than $\mu_{i}$ or (see (36) and (37)):

$$
\begin{aligned}
\varepsilon_{i}(w(\cdot))= & \mid \int_{0}^{\vartheta} 2\left(B^{\nu *} \psi_{i}^{*}(t), w(t)\right)_{U} \mathrm{~d} t \\
& -\int_{0}^{\vartheta} 2\left(B^{\nu *} \psi_{i}(t), w(t)\right)_{U} \mathrm{~d} t \mid \leq \mu_{i} .
\end{aligned}
$$

Using the Cauchy-Bunyakovsky inequality, we get

$$
\begin{aligned}
\varepsilon_{i}(w(\cdot)) \leq & \left(\int_{0}^{\vartheta}\left|B^{\nu}\right|_{L(U ; H)}^{2}|w(t)|_{U}^{2} \mathrm{~d} t\right)^{1 / 2} \\
& \times\left(\int_{0}^{\vartheta}\left|\psi_{i}^{*}(t)-\psi_{i}(t)\right|_{H}^{2} \mathrm{~d} t\right)^{1 / 2} \\
\leq & \left(|B|_{L(U ; H)}+\nu^{B}\right) C_{1} \varepsilon_{i}^{(1)} \vartheta^{1 / 2}=C_{2} \varepsilon_{i}^{(1)},
\end{aligned}
$$

where

$$
\varepsilon_{i}^{(1)}=\left|\psi_{i}^{*}(\cdot)-\psi_{i}(\cdot)\right|_{C(T, H)} .
$$

Therefore, for 477 it is sufficient to prove that

$$
\varepsilon_{i}^{(1)} \leq i \delta\left(\beta_{1}+C_{3} \beta_{2}\right)
$$

We prove the inequalities (48) by induction. Since $\psi_{i}^{*}(\cdot)=\psi_{i}(\cdot)=0$, the relation (48) holds for $i=0$. Assume that the relation holds for some $i$ and prove that

$$
\varepsilon_{i+1}^{(1)} \leq(i+1) \delta\left(\beta_{1}+C_{3} \beta_{2}\right)
$$

Due to (31) and 34, we have

$$
\left|\kappa_{i}^{*}(t)-\kappa_{i}(t)\right|_{H} \leq|G|_{L\left(H ; H_{1}\right)} \beta_{2}, \quad t \in T .
$$

Then, by virtue of (50), the solution $\bar{\zeta}_{i}(\cdot)$ of the Cauchy problem (30) solves the Cauchy problem

$$
\dot{\zeta}(t)=-A^{\nu *} \zeta(t)-G^{*} \kappa_{i}^{*}(t)+\lambda_{i}(t), \quad \zeta(\vartheta)=0,
$$


where

$$
\lambda_{i}(t)=G^{*}\left(\kappa_{i}(t)-\kappa_{i}^{*}(t)\right), \quad\left|\lambda_{i}(t)\right|_{H} \leq|G|_{L\left(H ; H_{1}\right)}^{2} \beta_{2} .
$$

Therefore, the function $\zeta_{i}(\cdot)=\bar{\zeta}_{i}(\cdot)-\zeta_{i}^{*}(\cdot)$ is a solution on $T$ of the Cauchy problem

$$
\dot{\zeta}(t)=-A^{\nu *} \zeta(t)+\lambda_{i}(t), \quad \zeta(\vartheta)=0 .
$$

Thus,

$$
\begin{aligned}
& \left|\bar{\zeta}_{i}(\cdot)-\zeta_{i}^{*}(\cdot)\right|_{C(T, H)} \\
& \quad \leq \vartheta \beta_{2}|G|_{L\left(H ; H_{1}\right)}^{2} \sup _{t \in T} \sup _{\nu \in[0,1]}\left|S^{\nu}(t)\right|_{L(H ; H)} \\
& \quad=C_{3} \beta_{2} .
\end{aligned}
$$

Hence, using (29), we derive

$$
\left|\zeta_{i}(\cdot)-\zeta_{i}^{*}(\cdot)\right|_{C(T ; H)} \leq \beta_{1}+C_{3} \beta_{2} .
$$

By virtue of (25) and (32), we conclude from (51) that

$$
\begin{aligned}
\varepsilon_{i+1}^{(1)} \leq \varepsilon_{i}^{(1)}+\delta \mid \zeta_{i}(\cdot)-\zeta_{i}^{*}(\cdot) & \left.\right|_{C(T ; H)} \\
& \leq \varepsilon_{i}^{(1)}+\delta\left(\beta_{1}+C_{3} \beta_{2}\right) .
\end{aligned}
$$

This implies (48).

Note that the inequalities

$$
\begin{aligned}
& \left|F u-F^{\nu} u\right|_{L_{2}\left(T ; H_{1}\right)} \\
& =\left(\int_{0}^{\vartheta}\left|\int_{0}^{\eta} G\left(S(\eta-t) B-S^{\nu}(\eta-t) B^{\nu}\right) u(t) \mathrm{d} t\right|_{H_{1}}^{2} \mathrm{~d} \eta\right)^{1 / 2} \\
& \leq \vartheta|G|_{L\left(H ; H_{1}\right)}\left\{\nu^{A}|B|_{L(U ; H)}+K_{3} \nu^{B}\right\} C_{1} \\
& \leq \nu^{F}=C_{4}\left(\nu^{A}+\nu^{B}\right),
\end{aligned}
$$

$$
\begin{aligned}
& \left|b^{\nu}-b\right|_{L_{2}\left(T ; H_{1}\right)} \\
& =\left(\int_{0}^{\vartheta}\left|G\left(S^{\nu}(\eta) x_{0}^{\nu}-S(\eta) x_{0}\right)\right|_{L\left(H ; H_{1}\right)}^{2} \mathrm{~d} \eta\right)^{1 / 2} \\
& \quad+\left(\int_{0}^{\vartheta}\left|\xi^{h}(t)-z(t)\right|_{H_{1}}^{2} \mathrm{~d} t\right)^{1 / 2} \\
& \quad \vartheta^{1 / 2}\left(|G|_{L\left(H ; H_{1}\right)}\left(K_{3} \nu^{x_{0}}+\left|x_{0}\right|_{H} \nu^{A}\right)+h\right) \\
& \leq \nu^{b}=C_{5}\left(\nu^{x_{0}}+\nu^{A}+h\right)
\end{aligned}
$$

are fulfilled. Here

$$
\begin{aligned}
& C_{4}=\vartheta|G|_{L\left(H ; H_{1}\right)} C_{1} \max \left\{K_{3},|B|_{L(U ; H)}\right\}, \\
& C_{5}=\vartheta^{1 / 2}\left(|G|_{L\left(H ; H_{1}\right)} \max \left\{K_{3},\left|x_{0}\right|_{H}\right\}+1\right) .
\end{aligned}
$$

The next statement follows from Theorems 1 and 2 together with Lemma 4.
Theorem 4. Let $\nu_{j}^{A} \rightarrow 0, \nu_{j}^{B} \rightarrow 0, \nu_{j}^{x_{0}} \rightarrow 0, h_{j} \rightarrow+0$, $\beta_{0, j} \rightarrow+0, \beta_{1, j} \rightarrow+0, \beta_{2, j} \rightarrow+0$ and Conditions (a)-(c) of Theorem 2, where $\nu_{j}^{F}=C_{4}\left(\nu_{j}^{A}+\nu_{j}^{B}\right), \nu_{j}^{b}=$ $C_{5}\left(\nu_{j}^{x_{0}}+\nu_{j}^{A}+h_{j}\right), \varepsilon_{j}=j C_{2} \delta_{j}\left(\beta_{1, j}+C_{3} \beta_{2, j}\right)+\beta_{0, j}$, be fulfilled. Let the sequences $\left\{y_{i}(\cdot), \psi_{i}(\cdot)\right\}_{i=0}^{j}$ be defined according to (24)-(31), where $\delta=\delta_{j}, \alpha=\alpha_{j}, h=h_{j}$, $\beta_{0}=\beta_{0, j}, \beta_{1}=\beta_{1, j}, \beta_{2}=\beta_{2, j}, \nu=\nu_{j}, \mid J^{\nu_{j}}(u(\cdot))-$ $J(u(\cdot)) \mid \leq \nu_{j}^{I} \equiv \nu_{j}^{J} \forall u(\cdot) \in U_{*}$. Then the sequence of elements $\left\{u_{j}\right\}_{j=1}^{+\infty}$,

$$
u_{j}=y_{j}(\cdot) /\left(\delta_{j} j\right),
$$

satisfies the conditions (9) and (10), i.e., it solves the problem of approximate determination of $u^{0}$ and $J^{0}$. In addition, the inequalities (b) of Theorem 1 are valid and any weakly convergent subsequence of the sequence $\left\{u_{j}\right\}_{j=1}^{+\infty}$ weakly converges in $L_{2}(T ; U)$ to the set $U_{0}(z)$.

Consequently from Theorem 4 and the inequalities 52), (53), the next statement follows.

Corollary 2. Let $j=j(h)=[1 / h], \nu_{j}^{A}=k^{(1)} h$, $\nu_{j}^{B}=k^{(2)} h, \nu_{j}^{x_{0}}=k^{(3)} h, \delta_{j}=h^{1-\varkappa},(\varkappa=\mathrm{const} \in$ $(0,1)), \alpha_{j}=h^{1 / 2-\varkappa / 2}, \beta_{1, j}=k^{(4)} h, \beta_{2, j}=k^{(5)} h$, $\beta_{0, j}=k^{(6)} h, \nu_{j}^{I}=k^{(7)} h$, and $h \in\left(0,1-\varepsilon_{*}\right)$, $\varepsilon_{*}=$ const $\in(0,1)$. Then the inequalities (19) and (20), in which $C$ and $C_{0}$ are some constants depending on $\varepsilon_{*}$,

$$
u^{h}=y_{j}(\cdot) /\left(h^{1-\varkappa}[1 / h]\right),
$$

are fulfilled. The element $y_{j}(\cdot)$ is defined according to (24)-(31) for $j=j(h)$

\section{Conclusions}

In the present work, the problem of source function reconstruction was under investigation. A new algorithm for solving such a problem for an abstract differential equation was suggested. This algorithm relies upon constructions of the theory of stable dynamical inversion based on the combination of methods of the theory of ill-posed problems and that of feedback control. The inversion theory exploits the idea of stabilizing appropriate functionals of the Lyapunov type by means of the extremal shift.

The work was supported by the Russian Foundation for Basic Research (project 10-01-00002), by the UralSiberian Project and by the program of the Presidium of the RAS Mathematical Control Theory.

\section{References}

Bensoussan, A., Prato, G.D., Delfour, M. and Mitter, S. (1992). Representation and Control of Infinite Dimensional Systems, Vol. I, Birkhäuser, Boston, MA.

Blizorukova, M.S. and Maksimov, V. I. (1998). On the reconstruction of an extremal input in a system with hereditary, Vestnik PGTU. Matematika i Prikladnaya Matematika (Mathematics and Applied Mathematics) 4(4): 51-61, (in Russian) 
Digas, B.V., Maksimov, V.I., Lander, A.V. and Bukchin, B.G. (2003). On an algorithm for solving the inverse problem of ray seismics, in D. Chowdhury (Ed.), Computational Seismology and Geodynamics, American Geophysical Union, Washington, DC, pp. 84-92.

Korbicz, J. and Zgurowski, M. (1991). Estimation and Control of Stochastic Distributed-Parameter Systems, Polish Scientific Publishers, Warsaw, (in Polish).

Krasovskii, N. and Subbotin, A. (1988). Game-Theoretical Control Problems, Springer, Berlin.

Kryazhimskii, A.V., Maksimov, V.I. and Osipov, Yu.S. (1997). Reconstruction of extremal disturbances in parabolic equations, Journal of Computational Mathematics and Mathematical Physics 37(3): 119-125, (in Russian).

Kryazhimskii, A.V. and Osipov, Yu.S. (1987). To a regularization of a convex extremal problem with inaccurately given constraints. An application to an optimal control problem with state constraints, in A.I. Korotkii and V.I. Maksimov (Eds.), Some Methods of Positional and Program Control, Ural Scientific Center, Sverdlovsk, pp. 34-54, (in Russian).

Omatu, S. and Seinfeld, J. (1989). Distributed Parameter Systems: Theory and Applications, Oxford Mathematical Monographs, Oxford University Press, New York, NY.
Uciński, D. (1999). Measurement Optimization for Parameter Estimation in Distributed Systems, Technical University Press, Zielona Góra.

Vasiliev, F. (1981). Solution Methods to Extremal Problems, Nauka, Moscow, (in Russian).

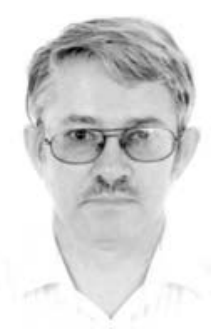

Vyacheslav Maksimov graduated in mathematics and mechanics from the Ural State University, Ekaterinburg, Russia, in 1972. He received the Candidate and the Doctor of Physics degrees from the Institute of Mathematics and Mechanics, Ural Branch of the Russian Academy of Sciences, in 1978 and 1992, respectively. Since 1972, he has been with the Institute of Mathematics and Mechanics, Ekaterinburg, Russia. Since 1994, he has been a department head at the same institute and a professor at the Chair of Controlled Systems Modeling of the Ural Federal University, Ekaterinburg, Russia. Since 2005, he has also been the head of the Chair. He is the author of more than 100 technical publications, including three monographs, and his research interests are primarily focused on control theory, distributed parameter systems, mathematical modeling. Dr. Maksimov is a member of the American Mathematical Society and IFIP WG7.2. He has been on editorial boards of various journals.

Received: 14 September 2009 Revised: 19 January 2010 